\title{
MODELO DE PREDICCIÓN PROBABILÍSTICA DE DETERIORO EN JAMÓN DE CERDO COCIDO
}

\author{
PROBABILISTIC PREDICTION MODEL OF DETERIORATION OF PORK COOKED HAM
}

\author{
Jhoan Sebastián LONDOÑO PARRA, Ing. Alimentos ${ }^{1}$; Kenneth Roy CABRERA TORRES, M.Sc. ${ }^{2}$; \\ María Isabel GONZÁLEZ HURTADO, M.Sc. ${ }^{\star \star}$
}

Recibido: Febrero 21 de 2017. Aprobado: Agosto 14 de 2018

\section{RESUMEN}

Antecedentes: el deterioro del jamón cocido es un proceso complejo que puede manifestarse por alteraciones de tipo microbiológico, fisicoquímico y sensorial, que hasta hoy han sido determinadas por metodologías tradicionales que incluyen largos periodos de seguimiento y un número importante de muestras. Objetivos: en el presente estudio se propuso un modelo para la predicción probabilística del deterioro de jamón cocido, por medio de análisis de componentes principales (ACP) y regresión logística con aproximación bayesiana. Métodos: para realizar el análisis, se evaluaron muestras provenientes de 300 lotes independientes de jamón de cerdo cocido, los cuales se conservaron en cámaras de almacenamiento a temperaturas de $12 \pm 1^{\circ} \mathrm{C}$. Se determinaron experimentalmente tres tipos de variables: fisicoquímicas ( $\mathrm{pH}$, sinéresis, nitritos residuales, porcentaje de ácido láctico), de textura (adhesividad, dureza, trabajo de punción y firmeza instrumental) y microbiológicas (mesófilos aerobios y bacterias acidolácticas) en dos tiempos de medición: 3 y 40 días. Para realizar la creación del modelo se seleccionaron aleatoriamente el $60 \%$ de los datos y para la validación el $40 \%$ restante. Los datos obtenidos fueron procesados con el paquete estadístico R Core Team 2012. Resultados: los nitritos residuales y la sinéresis fueron las variables más representativas, ya que su distribución, correlación y carga, fueron las más significativas, con mayor poder discriminante del fenómeno de deterioro en los dos momentos de medición. El modelo desarrollado permitió correlacionar los parámetros estudiados y predecir la probabilidad de deterioro del jamón, además de clasificarlo de acuerdo a su estado de calidad. La primera componente principal (CP1) (variables bioquímicas) explicó el 73,3 \% de la variación total de los datos, siendo los nitritos residuales y la sinéresis los factores más relacionados con el deterioro. Conclusión: el modelo logístico con aproximación bayesiana permitió obtener la probabilidad de deterioro del jamón cocido, almacenado a $12^{\circ} \mathrm{C}$ usando parámetros fisicoquímicos. La aplicación del ACP permitió correlacionar y clasificar los factores de deterioro del producto.

Palabras clave: Jamón Cocido, Deterioro de Alimentos, Predicción, Teorema de Bayes.

\begin{abstract}
Background: the spoilage of cooked ham is a complex process that may be manifested by alterations of microbiological, physicochemical and sensory type, which has been determined by traditional methodologies including long periods of monitoring with a significant number of samples. Objective: in the present study a model for probabilistic prediction of spoilage of cooked ham was proposed by principal component analysis (PCA) and logistic regression with Bayesian approach. Methods: to perform the analysis, independent samples from 300 batches of cooked pork ham were evaluated, which were stored in cold at temperatures $12 \pm 1^{\circ} \mathrm{C}$. Three types of variables were experimentally determined:

Centro de Investigación y Desarrollo Cárnico. Industria de Alimentos Zenú S.A.S. Grupo Nutresa. Medellín, Colombia.

Autor de correspondencia: mgonzalezh@zenu.com.co.
\end{abstract}


physicochemical ( $\mathrm{pH}$, syneresis, residual nitrite, lactic acid percentage), texture (adhesiveness, toughness, puncture and instrumental firmness) and microbiological (aerobic mesophilic bacteria and lactic acid bacteria) in two times: 3 and 40 days. To make this model were randomly selected $60 \%$ of the data and to validate it the remaining $40 \%$. The obtained data were processed with statistical package $\mathrm{R}$ package statistical Core Team 2012. Results: residual nitrite and syneresis were the most representative variables, since its distribution, correlation and loadings were the most significant, more discriminant of the phenomenon of spoilage in the two times of measurement. The developed model allowed correlating the parameters and predicting the spoilage probability of cooked ham, and classifying them according to their quality status. The first principal component (PC1) (biochemical variables) explained 73.3 $\%$ of the total variation, the residual nitrite and syneresis were the factors that related the spoilage. Conclusions: the logistic regression model with Bayesian approach allowed obtaining the probability of deterioration of cooked ham, stored at $12^{\circ} \mathrm{C}$ using physicochemical parameters. Applying the PCA permited to correlate and classify the factors of spoilage.

Keywords: Cooked ham, food spoilage, prediction, bayes theorem.

\section{INTRODUCCIÓN}

En productos cárnicos cocidos, durante el almacenamiento la matriz cárnica reacciona y pierde capacidad de retención de agua; esto ocasiona la generación de sinéresis que representa un riesgo mayor en cuanto a la calidad microbiológica ya que el agua disponible favorece la multiplicación de microorganismos presentes, alterando las características sensoriales con impacto en la vida útil (1-4). En jamón de cerdo cocido, algunos de los cambios que se producen por efecto de la sinéresis son: aparición de limosidad como resultado del crecimiento microbiano; acidez debida a la actividad metabólica bacteriana; y enverdecimiento debido a la producción de hidrógeno sulfurado por bacterias catalasa negativas (5).

La vida útil o durabilidad de un producto se define como el periodo de tiempo desde la fabricación en que éste mantiene una calidad global satisfactoria, evitando que llegue a ser sensorialmente inaceptable o que pueda suponer un riesgo para la salud del consumidor (6).

Hoy los métodos tradicionales para la determinación del tiempo de vida útil, incluyen el análisis de variables microbiológicas, fisicoquímicas y sensoriales a fin de determinar y entender dichos fenómenos. Estos métodos están siendo hoy reevaluados debido a que son costosos, requieren periodos de seguimiento extensos, un número importante de muestras y en ocasiones no alcanzan a explicar la dependencia entre variables; por esta razón hoy la industria cárnica tiene un enorme interés en la mejora y la eficiencia de las metodologías para la medición de la vida útil de productos cárnicos tipo jamón cocido, mediante la aplicación de técnicas estadísticas, que permitan analizar los cambios simultáneos y complejos que ocurren durante el tiempo de vida útil $(7,8)$. Uno de ellos es el propuesto por Pedro et al. (2006), quienes establecen que a partir de análisis multivariado es posible desarrollar modelos de predicción de deterioro en alimentos, basados en múltiples variables (9).

Escudero et al. (2014); mediante la determinación de parámetros microbiológicos, aplicaron el análisis estadístico multivariado como herramienta de control de calidad a fin de identificar puntos críticos de control durante el proceso de industrialización de carnes provenientes de aves de corral (10).

Mataragas et al. (2007), utilizó el análisis multivariado para modelar y predecir el deterioro de productos cárnicos cocidos y curados mediante análisis de diferentes parámetros como $\mathrm{pH}$, concentración de lactato, glucosa, acetato, recuento de BAL y mesófilos aerobios, a fin de encontrar correlaciones entre dichas variables y el deterioro mediante un análisis de clúster, ACP y regresión por mínimos cuadrados parciales (11).

Otro de los métodos empleados es el análisis de componentes principales (ACP), el cual agrupa las variables altamente correlacionadas que contienen información semejante, de manera que genera nuevas variables que expresan la información contenida en el conjunto original de datos y reduce la dimensionalidad del problema estudiado (12).

Valkova et al. (2007), utilizaron el análisis multivariado a través del ACP como indicador de calidad en el jamón cocido, mediante la correlación de parámetros instrumentales (perfil de textura, 
prueba de Warner-Bratzler y coordenadas de color: $\mathrm{L}^{\star} \mathrm{a}^{\star} \mathrm{b}$ ), sensoriales (aspecto de la superficie de corte, matriz, consistencia, color, olor, nivel de salado, gusto y terneza) y químicos (cantidad de materia, cenizas, cloruros, grasa, contenido de proteína) (13).

Cuando se tiene una variable respuesta binaria y se requiere estimar un modelo que prediga la probabilidad que alcance uno de los dos estados mediante variables explicativas se utiliza la regresión logística, en este caso se estima la incidencia de las variables explicativas sobre la probabilidad de adquirir uno de los valores binarios de la variable respuesta. Este tipo de regresión fue trabajada por Tabanelli et al. (2014), en la evaluación de dos agentes antimicrobianos frente al control de Sacharomyces cerevisiae, encontrando una región límite para el crecimiento o no crecimiento y con base en ello evaluar la interacción entre los agentes antimicrobianos (14).

La regresión logística también se ha utilizado favorablemente en la determinación de concentraciones de fagos apropiadas para la inhibición de Staphylococcus aureus en leches UHT, (15).

Tradicionalmente los modelos de regresión logística se ajustan utilizando la aproximación frecuentista, sin embargo en algunos casos no se puede estimar el ajuste debido a que son variables binarias y su comportamiento tiene una separación muy evidente, por lo tanto se requiere de aproximaciones bayesianas que permiten estimar adecuadamente los parámetros de la regresión (16)

Este tipo de metodologías han sido aplicadas en alimentos para modelar fenómenos de deterioro. Müller et al. (2006) evaluó parámetros de crecimiento microbiológico en salmón ahumado aplicando el enfoque bayesiano para el desarrollo de modelos de evaluación cuantitativa de riesgos microbiológicos (QMRA) (17).

Williams et al. (2010), encontraron que el método de síntesis bayesiano funciona bien en la evaluación de seguridad alimentaria de tipo microbiano al generar distribuciones de parámetros relevantes entre el efecto de Campylobacter en pollo, con relación a la estimación del número anual de enfermedades generadas por este microorganismo en un periodo de tiempo determinado (18). Pece et al. (2012); evaluaron la regresión logística en un estudio de plántulas de algarrobo blanco germinadas en vivero mediante diferentes concentraciones salinas; logrando obtener un modelo flexible de fácil interpretación de los parámetros estudiados; resultados a su vez acordes con lo reportado por López et al. (2011); al trabajar esta metodología con semillas de algodón (19, 20). Palacios et al. (2014); mediante estadística Bayesiana estudiaron un modelo de predicción de crecimiento de poblaciones microbianas múltiples con efectos complejos asociados a condiciones de producción industrial y de distribución logrando mejores predicciones para microorganismos patógenos como Lysteria sp que los métodos tradicionales (21)

Ranta et al. (2015), presentaron un método bayesiano para evaluar y comparar los efectos de criterios microbiológicos en la producción de pollos de engorde, con el cual pudieron analizar el riesgo predictivo del consumidor frente a la exposición de microorganismos específicos presentes en un lote de producción (22)

En el presente estudio se propuso un modelo para la predicción probabilística del deterioro de jamón cocido, por medio de análisis de componentes principales y regresión logística con aproximación Bayesiana con variables microbiológicas (microorganismos aerobios mesófilos y bacterias ácido lácticas) y físicoquímicas (sinéresis, $\mathrm{pH}$, acidez, nitrito residual) y físicas, (dureza, adhesividad, firmeza y punción) seleccionadas con base en investigaciones previas, ya que para la industria de alimentos, contar con herramientas que permitan tomar decisiones rápidas y confiables sobre el comportamiento y desempeño de sus productos, genera la oportunidad de dinamizar sus procesos de investigación y desarrollo, en función de acciones concretas fundamentadas en principios científicos, que impacten los resultados e incrementan el nivel de confianza por parte de los consumidores.

\section{MATERIALES Y MÉTODOS}

\section{Material de Estudio}

El jamón de cerdo cocido fue producido en la planta de Industria de Alimentos Zenú S.A.S (Medellín, Colombia) Grupo Nutresa, a partir de fórmulas estándar y con base en los protocolos de elaboración y envasado establecidos. Las muestras fueron empacadas por $450 \mathrm{~g}$ y almacenadas en cámaras de control térmico Thermo Scientific Model $815^{\circledR}$ a una temperatura de $12^{\circ} \mathrm{C} \pm 1^{\circ} \mathrm{C}$ que fue verificada con un sistema de adquisición de datos (Dataloger) utilizando el equipo Fluke 51 II. Los 
análisis se realizaron los días 3 y 40 respectivamente. El tamaño muestral fue de 300 paquetes de 450 g cada uno; cada paquete correspondía a un lote de producción diferente a fin de garantizar la independencia y la representatividad de la población de jamón. Los 300 paquetes se dividieron en dos tiempos de medición; 150 paquetes se analizaron al día 3 y los 150 restantes al día 40.

\section{Determinación del $\mathrm{pH}$}

Fue determinado utilizando un potenciómetro Oakton ION 2700 Benchtop Meter, previamente calibrado con soluciones amortiguadoras de $\mathrm{pH} 4,0$ y 7,0. Las mediciones se realizaron introduciendo la sonda en el interior del jamón, en tres puntos equidistantes de cada unidad muestral (paquete de $450 \mathrm{~g})$.

\section{Determinación de la sinéresis}

Se realizó por diferencia de peso a cada unidad muestral (paquete de $450 \mathrm{~g}$ ), en una balanza digital Metter Toledo PL 3001-S ${ }^{\circledR}$. Mediante la ecuación 1 (23).

Pérdida de agua $(g)=\frac{\text { Peso inicial }- \text { Peso final }}{\text { Peso inicial }}($ Ecuación 1$)$

\section{Determinación de la acidez}

Se analizó según NTC 4978, sobre $10 \mathrm{~g}$ de la unidad muestral homogenizada con $70 \mathrm{ml}$ de agua destilada, titulando con $\mathrm{NaOH} 0,1 \mathrm{~N}$ y usando fenolftaleína al $1 \%$ como indicador. El porcentaje de acidez, se calculó con base en la ecuación 2 (24).

$\%$ ácidoláctico $=$
$\frac{(V-V b)(N N a O H)(\text { meqácidoláctico })(f b)}{\text { Peso muestra }} \times 100$

(Ecuación 2)

Dónde:

- $\mathrm{V}=$ volumen de $\mathrm{NaOH}$ gastado en la muestra

- $\mathrm{Vb}=$ volumen de $\mathrm{NaOH}$ gastado en el blanco

- $\mathrm{N}=$ normalidad del $\mathrm{NaOH}$

- $\mathrm{fb}=$ factor de dilución

\section{Determinación de nitritos residuales}

Se realizó según el método de referencia descrito en NTC 4565. Se homogenizaron $5 \mathrm{~g}$ de la unidad muestral con $300 \mathrm{~mL}$ de agua destilada caliente a $80 \pm 1^{\circ} \mathrm{C}$, se transfirió una alícuota de $20 \mathrm{~mL}$ a un matraz de $50 \mathrm{~mL}$, se adicionaron $2,5 \mathrm{~mL}$ de reactivo de sulfanilamida y $2,5 \mathrm{~mL}$ del reactivo de
NED. Se determinó el porcentaje de tramitancia a una longitud de onda de $540 \mathrm{~nm}$. Se realizó ajuste del cero con un blanco conformado por $45 \mathrm{~mL}$ de agua destilada adicionados con $2,5 \mathrm{~mL}$ de reactivo de sulfanilamida y $2,5 \mathrm{~mL}$ de reactivo NED. La determinación del nitrito presente se realizó con base en la ecuación 3 (25).

$$
p p m=\frac{\% A x V(\text { total })}{m x D L x P M} \quad(\text { Ecuación } 3)
$$

Dónde:

- ppm $=$ partes por millón

- $\% \mathrm{~A}=\%$ de absorbancia

- $\mathrm{V}($ total $)=$ volumen total en $\mathrm{mL}(500 \mathrm{~mL})$

- $\mathrm{m}=$ pendiente de la curva de calibración

- $\mathrm{DL}=$ alícuota $(20 \mathrm{~mL})$

- $\mathrm{PM}=$ peso de la muestra en $\mathrm{g}$

\section{Determinación de firmeza (g.f) y trabajo de punción (g.sec).}

Se empleó un texturómetro universal TA.XT2i (Stable Micro Systems Ltd., Godalming, UK) provisto con una celda de carga de $30 \mathrm{Kg}$, una sonda de penetración múltiple (A/MPP; 12 pins de 12,2cm longitud x $0,4 \mathrm{~cm}$ de diámetro) y una plataforma de alta resistencia (HDP/90 Heavy Duty Platform). El test de textura se realizó sobre 5 tajadas de jamón pertenecientes a cada unidad experimental de $450 \mathrm{~g}$, las dimensiones de cada tajada eran de 10x10x1cm. Las condiciones de operación fueron: velocidad de pre-ensayo $1 \mathrm{~mm} / \mathrm{s}$, velocidad de ensayo $1 \mathrm{~mm} / \mathrm{s}$, velocidad de pos-ensayo $10 \mathrm{~mm} / \mathrm{s}$, nivel de penetración de $98 \%$. Se realizaron 5 repeticiones por muestra $(13,23,26$,).

\section{Determinación instrumental de adhesividad (g.f) y dureza (g.f).}

Se utilizó un texturómetro TA-XT2i (Stable Micro Systems Ltd., Godalming, UK) provisto con una celda de carga de $30 \mathrm{~kg}$ y una sonda de $75 \mathrm{~mm}$ de diámetro SMSP/75. El test de textura se realizó sobre 5 tajadas de jamón pertenecientes a cada unidad experimental de $450 \mathrm{~g}$, las dimensiones de cada tajada eran $10 \times 10 \times 0,2 \mathrm{~cm}$. Se comprimieron uniaxialmente un $75 \%$ de la altura original realizando 5 repeticiones por muestra. Las condiciones de operación fueron: velocidad de pre-ensayo $3 \mathrm{~mm} / \mathrm{s}$, tasa de compresión de $20 \% / \mathrm{s}$, velocidad pos-ensayo $0,8 \mathrm{~mm} / \mathrm{s}$ (13). 


\section{Determinaciones microbiológicas}

Para la realización de las pruebas microbiológicas (mesófilos y bacterias acido lácticas), se tomaron $10 \mathrm{~g}$ de cada unidad experimental que se diluyeron en $90 \mathrm{~mL}$ de agua peptonada; esta dilución se empleó posteriormente para la ejecución de cada determinación.

\section{Determinación de mesófilos aerobios}

Se determinaron a través del método NMP (Número más probable) usando el Kit TEMPO $\mathrm{TVC}^{\circledR}$, según el método oficial AOAC 966.23. La flora aerobia mesófila viable se incubó durante 40 a 48 horas. Se trabajó con tres diluciones y tres tubos por dilución. En el desarrollo de la técnica durante la incubación, los microorganismos presentes en la tarjeta degradan el sustrato del medio de cultivo causando la aparición de una señal fluorescente que detecta el lector (TEMPO Reader). En función del número y el tamaño de los pocillos positivos, se calculó el número de microorganismos presentes en la muestra original según lo establecido en el método NMP (Número Más Probable) (27).

\section{Determinación de bacterias acidolácticas (BAL)}

Se determinaron automáticamente a través del método incluído en el TEMPO LAB ${ }^{\circledR}$, con la tecnología TEMPO ${ }^{\circledR}$ (BioMéreux Industry), en el cual se obtienen niveles de prestación comparables con los de la NF ISO 15214, en 40-48 horas. Para el análisis, el TEMPO LAB $^{\circledR}$ consta de un frasco con un medio de cultivo y una tarjeta específica asociada. El medio inoculado se transfirió mediante una unidad de llenado (TEMPO Filler) a una tarjeta, que contiene 48 pocillos de tres volúmenes diferentes. La tarjeta contiene 3 grupos de 16 pocillos con un logaritmo de diferencia en volumen para cada grupo de pocillos, la cual simula el Número Más Probable (NMP). Los microorganismos presentes en la tarjeta reducen el substrato del medio de cultivo durante la incubación, y causan la aparición de una señal fluorescente, que es leída por el lector TEMPO ${ }^{\circledR}$. En función del número y del tamaño de los pocillos positivos, el sistema calcula automáticamente el número de microorganismo presentes en la muestra original de acuerdo con un cálculo basado en el método más probable (28).

\section{Análisis estadístico}

Los datos experimentales fueron analizados en el paquete estadístico R Core Team 2012. Para cada una de las variables, se hizo una comparación estadística entre los resultados obtenidos en los días 3 y 40 respectivamente, a fin de evidenciar estadísticamente cuales variables de manera individual discriminaban entre los tiempos de medición. Todas las variables estandarizadas se sometieron a un análisis de componentes principales (ACP), a fin de determinar las posibles correlaciones estadísticas entre ellas, el número de componentes más importante y las cargas de los coeficientes asociados con ellas. Una vez obtenidas las componentes principales, se seleccionaron las variables que presentaran un coeficiente más alto asociado a las componentes de mayor explicación. Estas variables se utilizaron en el ajuste de una regresión logística bayesiana. En este modelo se utilizó como variable de respuesta dicotómica el estado de "Deterioro" y "No Deterioro" de cada paquete. La estrategia Bayesiana se utilizó porque el modelo logístico tradicional presenta un sobreajuste y por lo tanto el modelo clásico no presenta convergencia para el estudio en cuestión. Dentro del enfoque Bayesiano, se seleccionó la distribución no informativa a priori (Cauchy). Para ajustar el modelo, se utilizó una muestra aleatoria del $60 \%$ de los datos y el 40 \% restante se utilizó para la validación del modelo. Los datos fueron procesados en el paquete estadístico R Core Team 2012.

\section{RESULTADOS}

\section{Estimación de componentes principales}

Se estimaron las funciones de densidad de probabilidad que representan las distribuciones de las variables para identificar la diferencia de los dos escenarios (deteriorado / no deteriorado) en los días de medición 3 y 40, mediante un suavizamiento tipo kernel. En la Figura 1, se presenta el comportamiento de las variables sinéresis, nitritos y $\mathrm{pH}$, que fueron las que mejor discriminaron el fenómeno de deterioro en las muestras de jamón de cerdo cocido; se observa que en el día 40 cuando se asume que el producto ya se encuentra deteriorado (línea roja) los nitritos presentan una menor dispersión y niveles más bajos, la sinéresis mayor dispersión y valores más altos y el pH mayor dispersión y valores más bajos. 

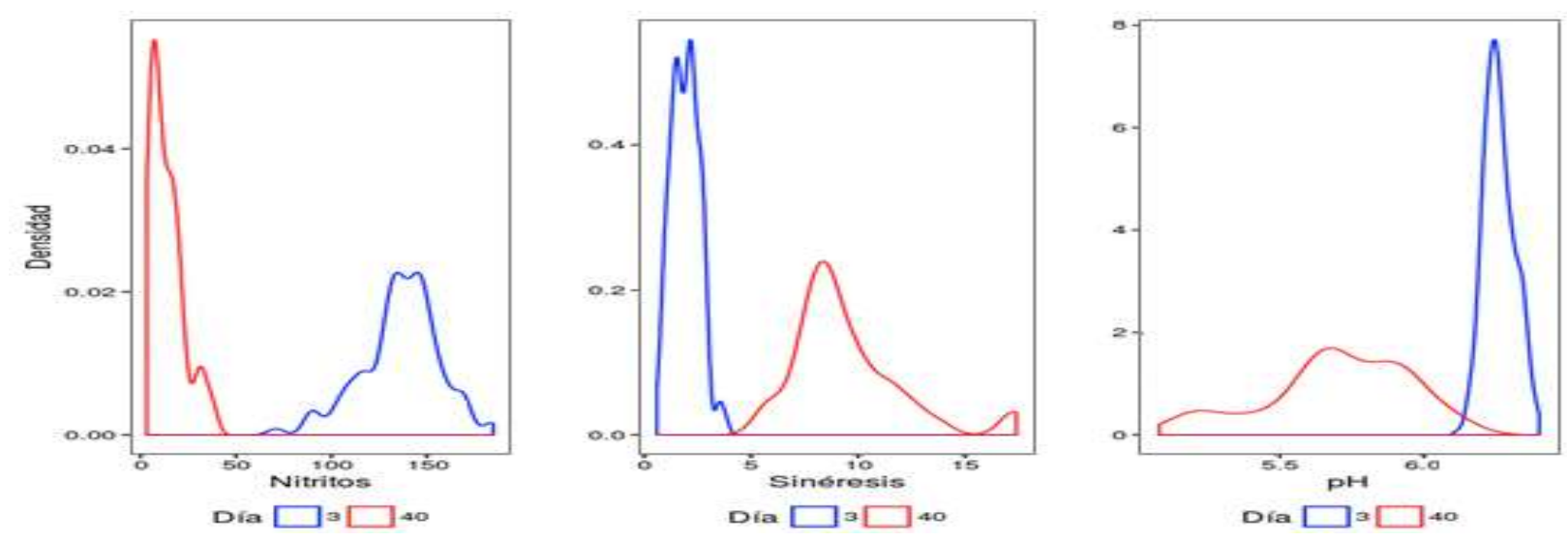

Figura 1. Estimación de la distribución de las variables: Nitritos, Sinéresis, pH. Día 3-, día 40—

En la Tabla 1, se evidencian las correlaciones de cada una de las variables. Se seleccionaron las variables que presentaron una alta correlación (superior al $80 \%$ ), para explicar el fenómeno de deterioro. Los símbolos menos y más " - / +", junto a los números indican una relación negativa o positiva entre dos variables. En el caso del nitrito, este se correlacionó negativamente con la sinéresis y el recuento de microorganismos aerobios mesófilos; y positivamente con el $\mathrm{pH}$. Cuando la sinéresis y el recuento de microorganismos aerobios mesófilos aumentaron, los nitritos disminuyeron, y cuando el valor del $\mathrm{pH}$ disminuyó el valor de los nitritos también lo hizo.

Tabla 1. Matriz de correlación de cada una de las variables explicativas.

\begin{tabular}{|l|c|c|c|c|c|c|c|c|c|c|}
\hline & SN & AL & $\mathbf{N}$ & L BAL & $\mathbf{L M}$ & $\mathbf{p H}$ & $\mathbf{F}$ & $\mathbf{P}$ & $\mathbf{D}$ & $\mathbf{A}$ \\
\hline SN & 1,0000 & 0,8229 & $-0,8845$ & 0,8605 & 0,8423 & $-0,8443$ & 0,7840 & 0,7281 & $-0,3856$ & $-0,5152$ \\
\hline AL & 0,8229 & 1,0000 & $-0,8418$ & 0,8191 & 0,7984 & $-0,8375$ & 0,7756 & 0,6793 & $-0,3469$ & $-0,5319$ \\
\hline N & $-0,8845$ & $-0,8418$ & 1,0000 & $-0,9114$ & $-0,9014$ & 0,9134 & $-0,8318$ & $-0,7727$ & 0,3993 & 0,5517 \\
\hline L BAL & 0,8605 & 0,8191 & $-0,9114$ & 1,0000 & 0,9682 & $-0,8990$ & 0,8289 & 0,7519 & $-0,4345$ & $-0,5253$ \\
\hline L M & 0,8423 & 0,7984 & $-0,9014$ & 0,9682 & 1,0000 & $-0,8574$ & 0,8236 & 0,7475 & $-0,4199$ & $-0,5021$ \\
\hline pH & $-0,8443$ & $-0,8375$ & 0,9134 & $-0,8990$ & $-0,8574$ & 1,0000 & $-0,8235$ & $-0,7485$ & 0,3724 & 0,5664 \\
\hline F & 0,7840 & 0,7756 & $-0,8318$ & 0,8299 & 0,8236 & $-0,8235$ & 1,0000 & 0,9333 & $-0,3468$ & $-0,4408$ \\
\hline P & 0,7281 & 0,6793 & $-0,7727$ & 0,7519 & 0,7475 & $-0,7485$ & 0,9333 & 1,0000 & $-0,3160$ & $-0,3769$ \\
\hline D & $-0,3856$ & $-0,3469$ & 0,3993 & $-0,4345$ & $-0,4199$ & 0,3724 & $-0,3468$ & $-0,3160$ & 1,0000 & 0,1893 \\
\hline A & $-0,5152$ & $-0,5319$ & 0,5517 & $-0,5253$ & $-0,5021$ & 0,5664 & $-0,4408$ & $-0,3769$ & 0,1893 & 1,0000 \\
\hline
\end{tabular}

SN (Sinéresis), AL (Ácido Láctico), N (Nitrito), LBAL (Log BAL), LM (Log Mesófilos), pH (pH), F (Firmeza), P (Punción), D (Dureza), A (Adhesividad)

En la Tabla 2, se presentan los coeficientes o cargas de las variables y el número de componentes principales (CP) del fenómeno. El primer componente (CP1), está asociado a las variables sinéresis, acidez láctica, nitritos, $\mathrm{pH}$, microorganismos aerobios mesófilos y bacterias acido lácticas; por ende se etiqueta como variables de comportamiento bioquímico. Se define el segundo componente (CP2) con la dureza y adhesividad y se etiqueta como variables de comportamiento físico.

Tomando como base lo reportado por Field (2000), las cargas con valores inferiores de 0.300 , se consideran no significativas, debido a que no tienen suficiente poder predictivo para el modelo porque son muy cercanas al cero, las cargas cercanas a uno, indican que las variables tienen una alta correlación y gran poder predictivo; es por esto que se escogieron los nitritos residuales y la sinéresis como las variables representativas, ya que su correlación, distribución y carga, además de ser las más altas $(0,8845)$, presentaron alto poder discriminante del fenómeno de deterioro en los dos momentos de medición (29). 
Tabla 2. Coeficientes de las variables y número de componentes principales.

\begin{tabular}{|l|c|c|c|c|c|c|c|c|c|c|}
\hline & CP1 & CP2 & CP3 & CP4 & CP5 & CP6 & CP7 & CP8 & CP9 & CP10 \\
\hline SN & 0,3380 & & & 0,2480 & 0,1840 & 0,8390 & $-0,1600$ & $-0,2400$ & & \\
\hline AL & 0,3290 & & & 0,2670 & 0,7390 & $-0,4070$ & $-0,2760$ & & 0,1420 & \\
\hline N & $-0,3530$ & & & $-0,1670$ & & $-0,1140$ & $-0,3020$ & $-0,8300$ & 0,1790 & $-0,1230$ \\
\hline L BAL & 0,3530 & & & 0,2300 & $-0,4010$ & $-0,1760$ & $-0,1540$ & $-0,2220$ & 0,1660 & 0,7280 \\
\hline L M & 0,3470 & & & 0,2270 & $-0,4880$ & $-0,1890$ & $-0,3860$ & & & $-0,6330$ \\
\hline pH & $-0,3470$ & & & $-0,1430$ & & 0,1520 & $-0,7870$ & 0,3930 & & 0,2180 \\
\hline F & 0,3360 & & $-0,3120$ & $-0,4070$ & & $-0,1310$ & & $-0,1810$ & $-0,7470$ & \\
\hline P & 0,3120 & & $-0,4080$ & $-0,5980$ & & & & 0,1140 & 0,5940 & \\
\hline D & $-0,1690$ & $-0,9220$ & $-0,2980$ & 0,1620 & & & & & & \\
\hline A & 0,2210 & 0,3630 & $-0,8020$ & 0,4060 & & & & & & \\
\hline
\end{tabular}

SN (Sinéresis), AL (Ácido Láctico), N (Nitrito), LBAL (Log BAL), LM (Log Mesófilos), pH (pH), F (Firmeza), P (Punción), D (Dureza), A (Adhesividad).

La Tabla 3, representa los componentes principales y su porcentaje de variación acumulado. En ella se evidencia que el componente principal 1 (CP1) explicó el 73,3\% de la variabilidad, mientras que el CP2 describe un 8,41\%. En conjunto explican un $81,71 \%$ de la variación total de los datos del fenómeno de deterioro. Esto implica que todas las variables están altamente correlacionadas entre sí, además es un grado muy alto de explicación de la variabilidad del fenómeno.

Tabla 3. Porcentaje de variación de cada componente principal.

\begin{tabular}{|c|c|}
\hline Componente (CP) & Porcentaje de Variación (\%) \\
\hline $\mathrm{CP} 1$ & 73,3 \\
\hline $\mathrm{CP} 2$ & 8,41 \\
\hline $\mathrm{CP} 3$ & 7,20 \\
\hline $\mathrm{CP} 4$ & 4,00 \\
\hline $\mathrm{CP} 5$ & 2,40 \\
\hline $\mathrm{CP} 6$ & 1,70 \\
\hline $\mathrm{CP} 7$ & 1,40 \\
\hline $\mathrm{CP} 8$ & 0,7 \\
\hline $\mathrm{CP} 9$ & 0,5 \\
\hline $\mathrm{CP} 10$ & 0,25 \\
\hline
\end{tabular}

Mataragas et al. (2007), al modelar y predecir el deterioro de productos cárnicos curados y cocidos mediante análisis multivariante encontró que un porcentaje de varianza acumulado por encima de $50 \%$ es adecuado para la realización de un modelo de tipo predictivo, este resultado es acorde con el presente estudio ya que los dos primeros componentes (CP1 y CP2) explican el $81,7 \%$ de la variación total (11), Así mismo, González et al. (2009), al estudiar la relación entre las características fisicoquímicas y sensoriales en jamón de cerdo cocido, encontraron que el análisis de componentes principales es un método muy útil para analizar la vinculación entre parámetros, al encontrar una correlación significativa entre el tiempo de almacenamiento y algunas variables fisicoquímicas como la sinéresis con un valor del $76 \%(23)$.

La Figura 2, representa en dos dimensiones, la distribución de las muestras en los componentes principales CP1 y CP2 respectivamente. El color corresponde al estado de conservación del producto siendo el color negro "No Deteriorado" y el color rojo "Deteriorado", esto permite ver que la componente principal CP1 discrimina claramente porque separa los dos grupos de las variables respuesta, uno a la izquierda y el otro a la derecha, siendo las variables bioquímicas las que logran discriminar el estado de deterioro del producto.

Se realizó verificación de los resultados de las componentes principales con la base de prueba, con el $60 \%$ de los datos y la verificación con el $40 \%$ restante. Se encontró que con el $40 \%$ se pudo predecir correctamente el $100 \%$ de las muestras restantes. 


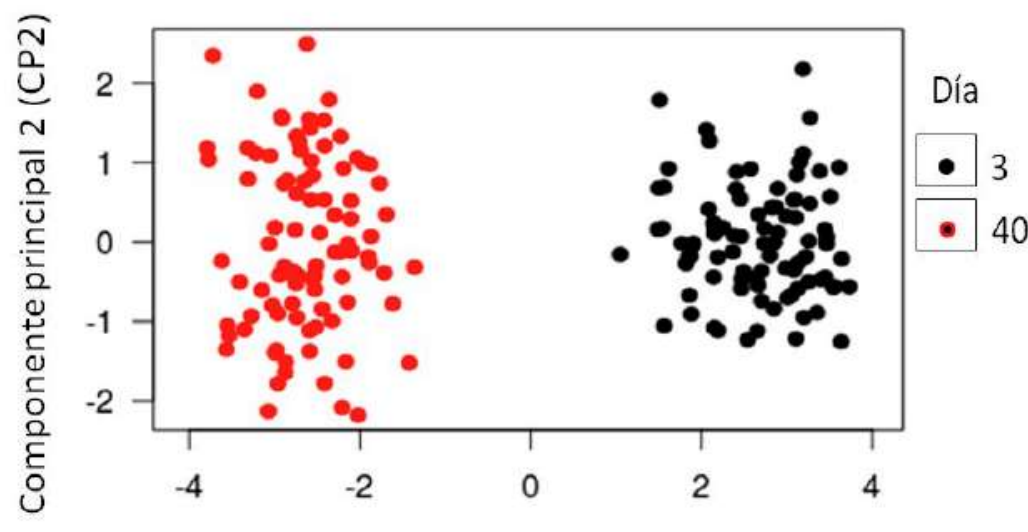

Componente principal 1 (CP1)

Figura 2. Componentes $\mathrm{CP} 1$ y CP2, $\bullet$ Producto no deteriorado, $\bigcirc$ Producto deteriorado.

\section{Modelo logístico con aproximación bayesiana}

Se construyó un modelo logístico con el fin de determinar la probabilidad de deterioro utilizando las variables asociadas al CP1 que mejor desempeño mostraron (nitritos y sinéresis)

Se estableció un rango de aceptación del 50\% (zona de estudio), como punto de referencia para clasificar las muestras en "deteriorado" (zona de rechazo) y "no deteriorado" (zona de aceptación). Este rango fue seleccionado debido a la complejidad que presenta el jamón en ser modelado con base al deterioro. La validación del modelo se realizó con el $40 \%$ de los datos escogidos aleatoriamente y es presentada en la Figura 3, donde se observa que los puntos verdes indican que los datos se encuentran en la zona de aceptación y pertenecen al grupo de las muestras medidas en el día 3, donde se evidenció que la concentración de nitrito residual fue alta y la cantidad de sinéresis baja; los puntos azules se ubicaron en la zona de rechazo y pertenecen al grupo de las muestras medidas en el día 40, en el cual la concentración de nitrito residual fue baja y la cantidad de sinéresis aumentó considerablemente. El modelo resultante se presenta en la Ecuación 4.

$$
p_{i}=\frac{1}{1-e^{\left.-\left(\beta_{0}+\beta_{1} x_{1}+\beta_{2} x_{2}\right)\right)}}
$$

(Ecuación 4)
Dónde:

$p_{i}=$ Probabilidad de estar deteriorado

$x_{1}=$ Nitrito

$x_{2}=$ Sinéresis

$\beta_{0}=14,6262$

$\beta_{1}=-0,5996$

$\beta_{2}=3,6606$

La Figura 3, representa la estimación de la probabilidad de deterioro mediante el modelo de estimación logístico bayesiano, el color rojo representa una probabilidad alta de deterioro, cercana a 1 y el color beige una probabilidad baja de deterioro cercana a 0 . El color amarillo representa el momento en el cual se hace la transición del estado de deterioro, sin embargo una vez se alcance el $50 \%$, se rechaza, los puntos verdes indican las unidades experimentales (verificación), evaluadas en el día 3 y que se encuentran en de la zona de aceptación. Los puntos azules hacen parte de la zona de rechazo y caracterizan al grupo de las unidades muestrales analizadas en el día 40. Las variables consideradas son la concentración de nitrito residual y la cantidad de sinéresis que muestran un comportamiento inverso entre sí; es decir, que las muestras con más alta sinéresis y un valor de nitrito residual bajo se clasifican como "Deterioradas", mientras que las muestras con alto recuento de nitritos y sinéresis baja corresponden al estado de "No deteriorado". 


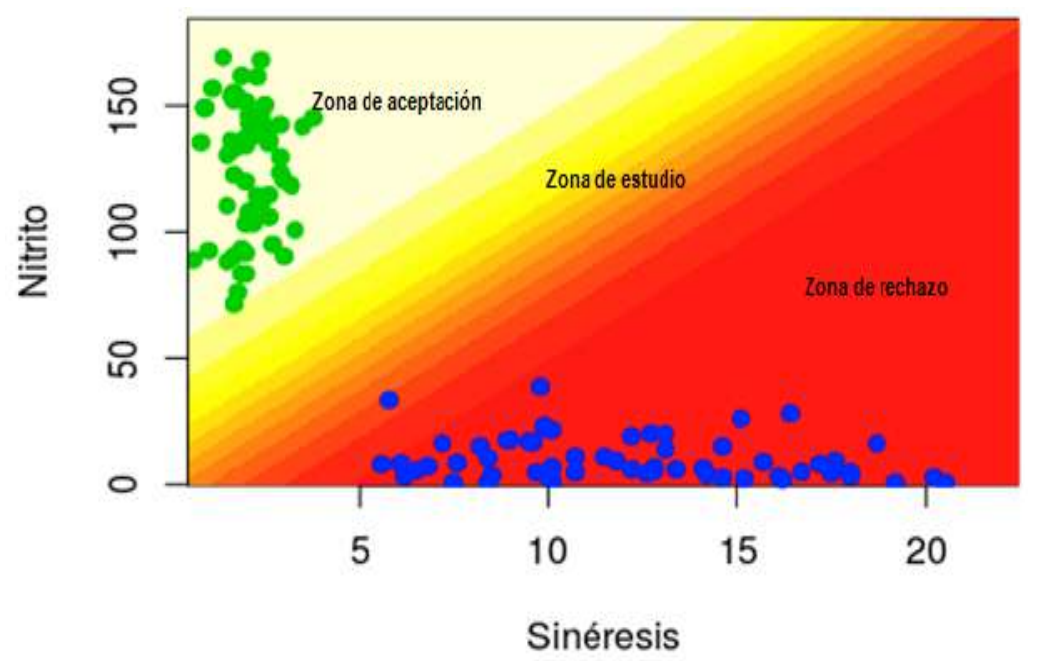

Figura 3. Modelo logístico con aproximación bayesiana.

\section{DISCUSIÓN}

\section{Estimación de los componentes principales}

Se encontró que existe una correlación negativa entre los nitritos y la sinéresis, ya que su comportamiento es inversamente proporcional a medida que transcurre el periodo de almacenamiento; este resultado es acorde a lo referenciado por Olafsdolttir et al. (2014) y Arnau y Casademont, (1987), en trabajos realizados con jamón cocido $(30,31)$. Se corrobora tal como lo establecen Mataragas et al. (2014), que la correlación existente entre las variables fisicoquímicas y microbiológicas es determinante en la clasificación del grado de deterioro de los productos cárnicos cocidos y definen su nivel de calidad (11)

En el ACP, el 73,3\% de la variación total de los datos del fenómeno de deterioro, fue explicado mediante la primer componente principal (CP1), equivalente al 73,3\%. Según Mataragas et al. (2007), un porcentaje de varianza acumulada y explicada por encima del $50 \%$ de la variación total en un componente principal, se considera satisfactorio para explicar el comportamiento del deterioro (11). En estudios desarrollados por Casiraghi et al. (2007), aplicaron el ACP para la clasificación de jamón cocido en base al nivel de inyección de salmuera y el origen de la carne de cerdo, generando una varianza acumulada de $56,4 \%$ y $76 \%$, respectivamente (32). El análisis de textura instrumental no presentó una discriminación del fenómeno, esto es acorde a lo reportado por Frontela et al. (2006), quienes encontraron que la textura medida de forma instrumental no es en general, un resultado que establezca diferencias que conlleven a la aceptación o rechazo en muestras de jamón cocido (33). En cuanto a la sinéresis, mostró un aumento a medida que transcurrió el periodo de almacenamiento, un comportamiento similar fue reportado por Gonzalez et al. (2010), en otro estudio realizado con jamón cocido (23).

\section{Modelo logístico con aproximación bayesiana}

A través del modelo logístico con aproximación bayesiana fue posible determinar la probabilidad de deterioro o no deterioro utilizando los datos obtenidos de sinéresis y nitritos residuales. Con base en la información descrita anteriormente es posible corroborar que herramientas estadísticas como ACP, en combinación con técnicas como las regresiones logísticas con aproximación bayesiana, pueden ser muy útiles para identificar variables relacionadas con la alteración, y construir modelos que estimen el estado de deterioro de alimentos como el jamón cocido; estos resultados presentan concordancia con lo reportado por Müller et al. (2006), al evaluar parámetros de crecimiento microbiológico en salmón ahumado aplicando el enfoque bayesiano para el desarrollo de modelos de evaluación cuantitativa de riesgos microbiológicos (17). Gelman et al. (2008) afirma que este tipo de modelos tiene una diferencia fundamental con respecto a otros modelos de predicción ya que la estimación de los parámetros tienen asociado un análisis estadístico de varianza estable dada la aproximación apriori que para este mismo autor corresponde a una distribución Cauchy (16). 


\section{CONCLUSIONES}

El modelo logístico con aproximación bayesiana permite obtener la predicción probabilística de deterioro del jamón cocido, almacenado a $12^{\circ} \mathrm{C}$ usando parámetros fisicoquímicos. La aplicación del ACP permite correlacionar y clasificar los factores de deterioro del producto, a través de un componente principal (CP1) que explica el 73,3\% de la variación total de los datos. Por medio de la correlación de los parámetros, se encontró que los nitritos residuales y la sinéresis fueron los factores más relacionados del deterioro; los cuales pueden ser utilizados como una forma de revelar indicadores que pueden ser útiles para la cuantificación de la calidad, así como para clasificar el grado de deterioro del jamón cocido.

\section{CONFLICTO DE INTERESES}

Los autores declaran que no existe conflicto de intereses en esta investigación.

\section{AGRADECIMIENTOS}

Los autores expresan sus agradecimientos por el apoyo financiero del presente estudio al Departamento Administrativo de Ciencia, Tecnología e Innovación "Colciencias" y al Centro de Investigación y Desarrollo del Negocio Cárnico del Grupo Nutresa.

\section{CONTRIBUCION DE LOS AUTORES}

Concepción y diseño del estudio: M. I. González H.; adquisición de datos: J. S. Londoño.; análisis de datos: K.R. Cabrera.

\section{LIMITACIONES DEL ESTUDIO}

Es de sumo interés utilizar esta misma metodología en otros productos cárnicos para estimar y cuantificar su deterioro durante el tiempo de vida útil, así como evaluar el producto en días intermedios para validar el modelo construído.

\section{BIBLIOGRAFÍA}

1. Li J Y, Yeh A I. Effects of starch properties on rheological characteristics of starch/meat complexes. J Food Eng [Internet]. 2003 May [cited 2014 Oct 3];57(3):287-94. Available from: http:// www.sciencedirect.com/science/article/pii/S0260877402003096.

2. Dexter DR, Sofos JN, Schmidt GR. Quality characteristics of turkey bologna formulated with carrageenan, starch, milk and soy protein. J Muscle Foods. 1993;4:207-23.
3. Pietrasik Z. Effect of content of protein, fat and modified starch on binding textural characteristics, and colour of comminuted scalded sausages. Meat Sci [Internet]. 1999 Jan [cited 2014 Oct 3]; 51(1):17-25. Available from: http://www.sciencedirect.com/ science/article/pii/S0309174098000680.

4. Carballo J, Barreto G, Jiménez Colmenero F. Starch and egg white influence on properties of bologna sausage as related to fat content. J Food Sci. 1996;60(4):673-7.

5. Castillo Castro C. Estudio de comportamiento de las propiedades microbiológicas y fisicoquímicas y organolépticas de productos cárnicos procesados a base de carne de pollo (salchichón y mortadela) durante el tiempo de vida útil estimada. Universidad de San Buenaventura; 2012.

6. Man D. Shelf Life. Food Ind Brief Ser. 2002;112.

7. Van Boekel MAJS. Kinetic modeling of food quality: A critical review. Comprehensive Reviews in Food Science and Food Safety. 2008. 144-58 p.

8. De Vleeschouwer K, Van der Plancken I, Van Loey A, Hendrickx ME. Modelling acrylamide changes in foods: from singleresponse empirical to multiresponse mechanistic approaches. Trends in Food Science and Technology. 2009. 155-67 p.

9. Pedro AMK, Ferreira MMC. Multivariate accelerated shelf-life testing: A novel approach for determining the shelf-life of foods. J Chemom. 2006;20(1-2):76-83.

10. Escudero-Gilete ML, González-Miret ML, Heredia FJ. Application of multivariate statistical analysis to quality control systems. Relevance of the stages in poultry meat production. Food Control [Internet]. 2014 Jun [cited 2015 Jun 30];40:243-9. Available from: http://www.sciencedirect.com/science/article/pii/ S0956713513006361.

11. Mataragas M, Skandamis P, Nychas GJE, Drosinos EH. Modeling and predicting spoilage of cooked, cured meat products by multivariate analysis. Meat Sci [Internet]. 2007 Nov [cited 2014 Oct 2];77(3):348-56. Available from: http://www.ncbi.nlm. nih.gov/pubmed/22061787.

12. Katsaounis TI. Methods of Multivariate Statistics. Technometrics. 2003. 100-1 p.

13. Válková V, Saláková a, Buchtová H, Tremlová B. Chemical, instrumental and sensory characteristics of cooked pork ham. Meat Sci [Internet]. 2007 Dec [cited 2014 Sep 26];77(4):608-15. Available from: http://www.ncbi.nlm.nih.gov/pubmed/22061949.

14. Tabanelli, G; Montanari, Chiara; Patrignani, Francesca; Gardini, Fausto. Modeling with the Logistic Regression of the Growth/ No Growth Interface of Saccharomyces cerevisiae in Relation to 2 Antimicrobial Terpenes (Citral and Linalool), $\mathrm{pH}$, and a(w), Journal of Food Science, 2014

15. Obeso JM, Garcia P, Martinez B, Arroyo-Lopez FN, GarridoFernandez A, Rodriguez A. Use of logistic regression for prediction of the fate of Staphylococcus aureus in pasteurized milk in the presence of two lytic phages. Appl Environ Microbiol 2010; 76:6038 - 6046; PMID: 20656872; http://dx.doi. org/10.1128/AEM.00613-10

16. Gelman, Andrew; Jakulin, Aleks; Grazia Pittau, Maria; Su, Yu-Sung. A Weakly Informative Default Prior Distribution For Logistic And Other Regression Models. The Annals of Applied Statistics, 2008, Vol. 2(4): 1360-1383

17. Muller ML, Cornu M, Pouillot R, Denis J-B. Use of Bayesian modelling in risk assessment: application to growth of Listeria monocytogenes and food flora in cold-smoked salmon. Int $\mathrm{J}$ Food Microbiol [Internet]. 2006 Mar 1 [cited 2015 Jun 30];106(2):195208. Available from: http://www.sciencedirect.com/science/ article/pii/S0168160505004332.

18. Williams NJ, Jones TR, Leatherbarrow HJ, Birtles RJ, LahuertaMarin L, Bennett M, Winstanley C. Isolation of a novel Campylobacter jejuni clone associated with the bank vole Myodes glareolus. Appl Environ Microbiol. 2010. 76: 7318-7321.

19. Pece MG, Acosta M, Saavedra S, Bruno C. Aplicación de la regresión logística en un estudio de emergencia de plántulas de 
algarrobo blanco (Prosopis alba Griseb.) en vivero, bajo diferentes concentraciones salinas. Quebracho. 2012. 20(1-2): 78-84

20. Lòpez FO. A Bayesian Approach to Parameter Estimation in Simplex Regression Model: A Comparison with Beta Regression. Revista Colombiana de Estadística. 2013. 36: 1-21.

21. Palacios AP, Marín JM, Quinto EJ, Wiper MP. Bayesian modeling of bacterial growth for multiple populations. Annals of Applied Statistics. (2014). 8(3), 1516-1537.

22. Ranta J, Lindgvist R, Hansson I, Tuominen P, Nauta W, et al. A Bayesian approach to the evaluation of risk-based microbiologial criteria for Campylobacter in broiler meat. The Annals of Applied Statistics. 2015. 9(3): 1415-1432.

23. Gonzalez MI, Suárez H, Martínez O. Influencia del proceso de cocción y temperatura de almacenamiento sobre las características fisicoquímicas, microbiológicas y sensoriales del jamón de cerdo [Internet]. Revista Colombiana de Ciencias Pecuarias (Colombian journal of animal science and veterinary medicine). 2010 [cited 2015 Jun 9]. 336-48 p. Available from: http://rccp. udea.edu.co/index.php/ojs/article/view/624/560.

24. Instituto Colombiano de Normas Técnicas y Certificación (ICONTEC). Norma Técnica Colombiana NTC 4978 [Internet]. 2001, 11 p. Available from: www.icontec.org

25. Instituto Colombiano de Normas Técnicas y Certificación (ICONTEC). Norma Técnica Colombiana NTC 4565 [Internet]. 2010, 13 p. Available from: www.icontec.org.

26. Cheng Q, Sun DW. Quality of pork ham as affected by locations within sample. Cooking methods and storage. J Food Eng. 2004; 65(4): 551-556.

27. Association of Official Analytical Chemist (AOAC). Official Methods of Analysis of AOAC International (Method
966.23/1989). Assoc Off Anal Chem Int [Internet]. 2012; CDROM. Available from: www.aoac.org.

28. ISO-International Organization for Standardization. Microbiologie des aliments -- Méthode horizontale pour le dénombrement des bactéries lactiques mésophiles. NF ISO 15214 [Internet]. ISO/TC 34/SC 9. 1998, 7 p. Available from: www. iso.org.

29. Field AP. Discovering Statistics Using SPSS for Windows: Advanced Techniques for Beginners. Sage Publications, Inc.; 2000 Jul 1 [cited 2015 May 21]; Available from: http://dl.acm. org/citation.cfm?id=518130.

30. Olafsdottir G, Lauzon HL, Martinsdottir E, Kristbergsson $\mathrm{K}$. Influence of storage temperature on microbial spoilage characteristics of haddock fillets (Melanogrammus aeglefinus) evaluated by multivariate quality prediction. Int J Food Microbiol [Internet]. 2006 Sep 1 [cited 2014 Dec 21];111(2):112-25. Available from: http://www.sciencedirect.com/science/article/ pii/S0168160506002662

31. Arnau J, Casademont G. El envasado del jamón. Capítulo I: Parámetros fisicoquímicos en jamón curado deshuesado y envasado al vacío. Girona, Spain; 1987.

32. Casiraghi E, Alamprese C, Pompei C. Cooked ham classification on the basis of brine injection level and pork breeding country. LWT - Food Sci Technol [Internet]. 2007 Jan [cited 2015 Jul 1]; 40(1): 164-9. Available from: http://www.sciencedirect.com/ science/article/pii/S0023643805001593

33. Frontela C, López G, Ros G, Martínez C. Relación entre los parámetros sensoriales, físicoquímicos e instrumentales en el jamón cocido. An Vet Murcia. 2006; 22: 67-78. 